Volume 353, Number 10, Pages 4155-4171

S 0002-9947(01)02765-9

Article electronically published on May 17, 2001

\title{
ON THE INVERSE SPECTRAL THEORY OF SCHRÖDINGER AND DIRAC OPERATORS
}

\author{
MIKLÓS HORVÁTH
}

\begin{abstract}
We prove that under some conditions finitely many partially known spectra and partial information on the potential entirely determine the potential. This extends former results of Hochstadt, Lieberman, Gesztesy, Simon and others.
\end{abstract}

\section{INTRODUCTION}

Consider the Schrödinger operator

$$
-y^{\prime \prime}+q(x) y=\lambda y \quad \text { on } \quad(0, \pi)
$$

with the boundary conditions

$$
\begin{aligned}
& y(0) \cos \alpha+y^{\prime}(0) \sin \alpha=0, \\
& y(\pi) \cos \beta+y^{\prime}(\pi) \sin \beta=0,
\end{aligned}
$$

where $q \in L_{1}(0, \pi)$. The eigenvalues of (1.1)-(1.3) form the sequence

$$
\lambda_{0}<\lambda_{1}<\ldots
$$

Given a complex value $\lambda$ define $y(x, \lambda)$ as the solution of (1.1) with the initial conditions

$$
y(0)=\sin \alpha, \quad y^{\prime}(0)=-\cos \alpha .
$$

For $\lambda=\lambda_{n}, y\left(x, \lambda_{n}\right)$ is the eigenfunction of (1.1)-(1.3) corresponding to the eigenvalue $\lambda_{n}$. Introduce the normalizing constants

$$
\alpha_{n}^{2}=\int_{0}^{\pi} y^{2}\left(x, \lambda_{n}\right) \mathrm{d} x
$$

The spectral function of the problem (1.1)-(1.3) is defined to be

$$
\varrho(\lambda)=\left\{\begin{array}{cccc}
\sum_{0<\lambda_{n} \leqslant \lambda} & \frac{1}{\alpha_{n}^{2}} \quad \text { if } \quad \lambda>0, \\
-\sum_{\lambda<\lambda_{n} \leqslant 0} & \frac{1}{\alpha_{n}^{2}} \quad \text { if } \quad \lambda<0 .
\end{array}\right.
$$

Received by the editors February 16, 2000 and, in revised form, June 7, 2000.

1991 Mathematics Subject Classification. Primary 34A55, 34B20; Secondary 34L40, 47A75.

Key words and phrases. Inverse spectral theory, $m$-function, spectral function.

Research supported by the Hungarian NSF Grant OTKA T\#32374. 
A fundamental result of Marchenko [12] says that the spectral function determines the potential. A similar statement was given by G. Borg [2] on the Titchmarsh-Weyl m-function of the problem (1.1)-(1.3) as defined by

$$
m(\lambda)=\frac{v^{\prime}(0, \lambda)}{v(0, \lambda)}, \quad \lambda \in \mathbb{C},
$$

where $v(x, \lambda)$ is the solution of (1.1) with the initial conditions

$$
v(\pi, \lambda)=\sin \beta, \quad v^{\prime}(\pi, \lambda)=-\cos \beta .
$$

More precisely, let $q^{*}(x)$ be another potential and denote $\varrho^{*}$ and $m^{*}$ the corresponding functions. Then the two results together can be formulated as

Theorem $1.1([2],[12])$. a) If $\varrho^{*}(\lambda)=\varrho(\lambda)$ for $\lambda \in \mathbb{R}$, then $q^{*}(x)=q(x)$ a.e. in $(0, \pi)$.

b) If $m^{*}(\lambda)=m(\lambda)$ for $\lambda \in \mathbb{C} \backslash \mathbb{R}$, then $q^{*}=q$ a.e. in $(0, \pi)$.

The two statements are connected since $m$ and $\varrho$ can be expressed from each other (see e.g. 9]).

Now consider the eigenvalue problem corresponding to the Dirac operator

$$
\begin{aligned}
u_{2}^{\prime}+(V(x)+\mu) u_{1} & =\lambda u_{1} \quad \text { on } \quad(0, \pi) \\
-u_{1}^{\prime}+(V(x)-\mu) u_{2} & =\lambda u_{2}
\end{aligned}
$$

with the boundary conditions

$$
\begin{aligned}
u_{1}(0) \cos \alpha+u_{2}(0) \sin \alpha & =0, \\
u_{1}(\pi) \cos \beta+u_{2}(\pi) \sin \beta & =0 .
\end{aligned}
$$

Here we suppose that the potential $V(x)$ is continuous on $[0, \pi]$ and the mass $\mu$ is positive. The Dirac operator is the relativistic Schrödinger operator in quantum physics. The spectral function of the problem (1.8)-(1.10) is again defined by (1.5) with

$$
\alpha_{n}^{2}=\int_{0}^{\pi}\left[u_{n, 1}^{2}\left(x, \lambda_{n}\right)+u_{n, 2}^{2}\left(x, \lambda_{n}\right)\right] \mathrm{d} x, \quad n \in \mathbb{Z},
$$

where $\left(u_{n, 1}(x, \lambda), u_{n, 2}(x, \lambda)\right)$ is the solution of (1.8) with the initial conditions

$$
u_{n, 1}(0, \lambda)=\sin \alpha, u_{n, 2}(0, \lambda)=-\cos \alpha .
$$

The relativistic version of Theorem 1.1

Theorem 1.2 (9], 13]). Let $\varrho^{*}$ be the spectral function corresponding to the data $V^{*}, \mu^{*}, \alpha^{*}$ and $\beta^{*}$ instead of $V, \mu, \alpha$ and $\beta$. If $\varrho^{*}=\varrho$, then $V^{*}=V, \mu^{*}=$ $\mu, \tan \alpha^{*}=\tan \alpha, \tan \beta^{*}=\tan \beta$.

Furthermore define the $m$-function as

$$
m(\lambda)=\frac{v_{2}(0, \lambda)}{v_{1}(0, \lambda)},
$$

where $\left(v_{1}(x, \lambda), v_{2}(x, \lambda)\right)$ is the solution of $(1.8)$ with the initial conditions

$$
v_{1}(\pi, \lambda)=\sin \beta, v_{2}(\pi, \lambda)=-\cos \beta .
$$

Establishing the appropriate equation connecting $\varrho$ and $m$, we get

Theorem 1.3. If $m^{*}(\lambda)=m(\lambda)$, then $V^{*}=V, \mu^{*}=\mu$ and $\tan \beta^{*}=\tan \beta$. 
Returning to the Schrödinger operator, it is known from Borg [1] that two spectra (defined by the boundary conditions (1.2)-(1.3) with $\alpha_{1}, \beta$ and $\alpha_{2}, \beta$ ) determine the potential $q$. Hochstadt and Lieberman [7] proved that if half of the potential is known, then one spectrum is enough:

Theorem $1.4([7])$. Suppose that $\cos \alpha \neq 0$ and that $q^{*}=q$ a.e. on $\left(0, \frac{\pi}{2}\right)$. If the spectrum of (1.1)-(1.3) of $q$ and of $q^{*}$ is identical, then $q^{*}=q$ a.e. on the whole $(0, \pi)$.

Gesztesy and Simon 5] showed that more information on the potential can compensate for less information on the spectrum in the following sense:

Theorem $1.5([5])$. Let $0<\gamma<1$ and suppose that $q^{*}=q$ a.e. on $\left(0, \frac{1+\gamma}{2} \pi\right)$, $\tan \beta^{*}$ and $\tan \beta \neq \infty$, $\tan \alpha^{*}=\tan \alpha \neq \infty$. Denote $S=\left\{\lambda_{n}: \lambda_{n}^{*}=\lambda_{n}\right\}$. If

$$
\#\left\{\lambda \in S: \lambda \leqslant t_{0}\right\} \geqslant(1-\gamma) \#\left\{\lambda_{n}: \lambda_{n}<t_{0}\right\}+\alpha / 2
$$

for all sufficiently large $t_{0}$, then $\tan \beta^{*}=\tan \beta$ and $q^{*}=q$ a.e. on $(0, \pi)$.

Later on del Rio, Gesztesy and Simon found the following related statements (see also [4]):

Theorem 1.6 ([3]). Let $\sigma_{N}$ and $\sigma_{D}$ be the spectrum of (1.1)-(1.3) with $\alpha=0$, respectively $\alpha=\frac{\pi}{2}$. Let $S_{N} \subset \sigma_{N}, S_{D} \subset \sigma_{D}$ and $0<\gamma<1$. If

$$
\#\left\{\lambda \in S_{N} \cup S_{D}: \lambda \leqslant t_{0}\right\} \geqslant(1-\gamma) \#\left\{\lambda \in \sigma_{N} \cup \sigma_{D}: \lambda \leqslant t_{0}\right\}
$$

holds for all sufficiently large $t_{0}$, then $S_{N}, S_{D}$ and $q$ on $(0, \gamma \pi)$ determine $q$ a.e. on $(0, \pi)$.

Theorem 1.7 ([3]). Let $\sigma_{1}, \sigma_{2}, \sigma_{3}$ be the spectrum of (1.1)-(1.3) with $\alpha_{1}, \alpha_{2}, \alpha_{3}$ in (1.2). Let $S_{j} \subset \sigma_{j}$ satisfying

$$
\begin{aligned}
\#\{\lambda & \left.\in S_{1} \cup S_{2} \cup S_{3}: \lambda \leqslant t_{0}\right\} \\
& \geqslant \frac{2}{3} \#\left\{\lambda \in \sigma_{1} \cup \sigma_{2} \cup \sigma_{3}: \lambda \leqslant t_{0}\right\}
\end{aligned}
$$

for all sufficiently large $t_{0}$. Then $S_{1}, S_{2}, S_{3}$ determine $q$ a.e. on $(0, \pi)$.

We will prove the following generalization of the above-mentioned result of Borg and Theorems 1.4-1.7 (formulated on the segment $(0, \pi)$ ):

Theorem 1.8. Let $\sigma_{j}, j=1, \ldots, N$, be the spectrum of (1.1)-(1.3) with $\alpha_{j}$ in (1.2). Suppose that $\lambda_{n}^{(j)} \in \sigma_{j}$ is known for $n \in S_{j}$ and denote

$$
n_{j}(t)=\sum_{\lambda_{n}^{(j)}<t^{2}, n \in S_{j}} 1
$$

for $t \geqslant 0$. Let $0 \leqslant a<\pi, 0 \leqslant \gamma \leqslant 1$, and suppose that there exist $t_{0}>0$ and $\delta>0$ such that for $t \geqslant t_{0}$

$$
\sum_{j=1}^{N} n_{j}(t) \geqslant\left\{\begin{array}{r}
2\left(1-\frac{a}{\pi}\right)\left\{\gamma\left[t+\frac{1}{2}\right]+(1-\gamma)\left([t]+\frac{1}{2}\right)\right\}+ \\
\mathbf{O}\left(t^{-\delta}\right) \\
\text { if } \sin \beta \neq 0 \\
2\left(1-\frac{a}{\pi}\right)\left\{\gamma\left[t+\frac{1}{2}\right]+(1-\gamma)\left([t]+\frac{1}{2}\right)\right\}-1+\mathbf{O}\left(t^{-\delta}\right) \\
\text { if } \sin \beta=0
\end{array}\right.
$$

Then $q$ on $(0, a)$ and the eigenvalues $\left\{\lambda_{n}^{(j)}: n \in S_{j}\right\}, j=1, \ldots, N$, determine q a.e. on $(0, \pi)$. 
The counterpart for the Dirac case reads as follows:

Theorem 1.9. Let $\sigma_{j}, j=1 \ldots, N$, be the spectrum of (1.8)-(1.10) with $\alpha_{j}$ in (1.9). Suppose that $\lambda_{n}^{(j)} \in \sigma_{j}$ is known for $n \in S_{j}$ and denote

$$
\tilde{n}_{j}(t)=\left\{\begin{array}{ccc}
\sum_{0<n<t, n \in S_{j}} 1 & \text { if } t>0, \\
-\sum_{t<n<0, n \in S_{j}} 1 & \text { if } t<0 .
\end{array}\right.
$$

(Thus, we will not count the value $n=0$.) Suppose that the sets $S_{j}$ are almost symmetrical, i.e. that $n \in S_{j}$ implies $-n \in S_{j}$ with finitely many possible exceptions and that the limits

$$
\lim _{t \rightarrow \infty} \frac{\widetilde{n}_{j}(t)}{t}=\gamma_{j}
$$

exist for $j=1, \ldots, N$. Denote by $d$ the number of indices $j$ with $0 \in S_{j}$.

Let $\mu \in \mathbb{R}, 0 \leqslant a<\pi, t_{0}>0, \delta>0$ and $\varepsilon>0$ be arbitrary numbers. Suppose finally that

$$
\sum_{j=1}^{N} \widetilde{n}_{j}(t)\left\{\begin{array}{r}
\geqslant 2\left(1-\frac{a}{\pi}\right)[t]-d+\mu-1+\varepsilon+\mathbf{O}\left(t^{-\delta}\right) \\
\text { if } t \geqslant t_{0}, \\
\leqslant-2\left(1-\frac{a}{\pi}\right)[-t]+\mu-2\left(1-\frac{a}{\pi}\right)+\mathbf{O}\left(|t|^{-\delta}\right) \\
\text { if } t \leqslant-t_{0} .
\end{array}\right.
$$

Then $V$ on $[0, a]$ and the eigenvalues $\left\{\lambda_{n}^{(j)}: n \in S_{j}\right\}, j=1, \ldots, N$, determine $V$ on $[0, \pi]$.

\section{Partial information on the Potential}

In this section we will prove Theorems 1.3, 1.8 and 1.9. Though Theorem 1.3 might exist somewhere in the literature, I was not able to find it, so I will provide a proof. In order to do so, we need the Green function (or Green matrix) of the problem (1.8)-(1.10). It has the form

$$
G(x, y, \lambda)=\text { p.v. } \sum_{-\infty}^{\infty} \frac{u\left(x, \lambda_{n}\right) u^{T}\left(y, \lambda_{n}\right)}{\alpha_{n}^{2}\left(\lambda-\lambda_{n}\right)}, \quad \lambda \neq \lambda_{n} .
$$

where $u=\left(\begin{array}{l}u_{1} \\ u_{2}\end{array}\right)$, p.v. $\sum=\lim _{N \rightarrow \infty} \sum_{-N}^{N}$. The sum is convergent at every point $(x, y)$ and the convergence is locally uniform outside the diagonal $x=y$. Consequently the Green matrix is continuous for $x<y$ and $x>y$, and the sum can be expressed in closed form (see e.g. Levitan, Sargsjan [10]):

$$
G(x, y, \lambda)= \begin{cases}\frac{-1}{W(\lambda)} u(x, \lambda) v^{T}(y, \lambda) & \text { if } x<y, \\ \frac{-1}{W(\lambda)} v(x, \lambda) u^{T}(y, \lambda) & \text { if } y<x .\end{cases}
$$

Here $v=\left(\begin{array}{l}v_{1} \\ v_{2}\end{array}\right)$ and

$$
W(\lambda)=u_{1}(x, \lambda) v_{2}(x, \lambda)-u_{2}(x, \lambda) v_{1}(x, \lambda) .
$$

This function $W$ is independent of $x$ since

$$
\begin{aligned}
W^{\prime}= & u_{1}^{\prime} v_{2}-u_{2}^{\prime} v_{1}+u_{1} v_{2}^{\prime}-u_{2} v_{1}^{\prime}=(V-m-\lambda) u_{2} v_{2} \\
& -(\lambda-V-m) u_{1} v_{1}+(\lambda-V-m) u_{1} v_{1}-(V-m-\lambda) u_{2} v_{2}=0 .
\end{aligned}
$$


Lemma 2.1.

$$
\begin{aligned}
G_{11}(0,0, \lambda) & =\sin ^{2} \alpha \cdot p . v \cdot \int_{-\infty}^{\infty} \frac{d \rho(t)}{\lambda-t} \\
& =\sin \alpha \cos \alpha-\frac{\sin \alpha}{m(\lambda) \sin \alpha+\cos \alpha}
\end{aligned}
$$

where $G_{11}$ is the left upper element of the matrix $G$ and p.v. $\int_{-\infty}^{\infty}=\lim _{R \rightarrow \infty} \int_{-R}^{R}$.

Proof. We are looking for the value of the jump

$$
G_{11}(0,0+0, \lambda)-G_{11}(0,0, \lambda)
$$

By definition

$$
G_{11}(0, y, \lambda)=\sin \alpha \cdot \text { p.v. } \sum \frac{u_{1}\left(y, \lambda_{n}\right)}{\alpha_{n}^{2}\left(\lambda-\lambda_{n}\right)} .
$$

Recall the asymptotical formulae

$$
\begin{gathered}
u_{1}(x, \lambda)=\sin \left(\lambda x-\int_{0}^{x} V+\alpha\right)+\mathbf{O}\left(\frac{e^{|\Im \lambda| x}}{|\lambda|}\right), \\
u_{2}(x, \lambda)=-\cos \left(\lambda x-\int_{0}^{x} V+\alpha\right)+\mathbf{O}\left(\frac{e^{|\Im \lambda| x}}{|\lambda|}\right), \quad|\lambda| \rightarrow \infty,
\end{gathered}
$$

with remainder terms uniform in $x$ and

$$
\alpha_{n}^{2}=\pi+\mathbf{O}\left(\frac{1}{1+|n|}\right)
$$

see [9]. Since $\alpha_{n}^{2}\left(\lambda-\lambda_{n}\right)=-\pi n+\mathbf{O}(1)$, we see that $G_{11}$ has the same jump as

$$
\sin \alpha \cdot \text { p.v. } \sum_{n \neq 0} \frac{\sin \left(\lambda_{n} y-\int_{0}^{y} V+\alpha\right)}{-\pi n}
$$

(because the difference is continuous). Now consider the eigenvalue asymptotics:

$$
\lambda_{n}=n+\frac{\vartheta}{\pi}+\mathbf{O}\left(\frac{1}{1+|n|}\right), \quad \vartheta=\beta-\alpha+\int_{0}^{\pi} V .
$$

We get

$$
\begin{aligned}
\sin \left(\lambda_{n} y-\int_{0}^{y} V+\alpha\right) & =\sin \left(n y+a(y)+\mathbf{O}\left(\frac{1}{1+|n|}\right)\right), \\
a(y) & =\frac{\vartheta}{\pi} y-\int_{0}^{y} V+\alpha .
\end{aligned}
$$

So (2.5) has the same jump as

$$
\begin{aligned}
\sin \alpha \cdot \text { p.v. } \sum_{n \neq 0} \frac{\sin (n y+a(y))}{-\pi n} \\
=\sin \alpha \cdot \cos a(y) \cdot \frac{2}{-\pi} \sum_{n=1}^{\infty} \frac{\sin n y}{n} .
\end{aligned}
$$


It is known that $\sum_{n=1}^{\infty} \frac{\sin n y}{n}$ is the Fourier series of $\frac{\pi-y}{2}$ on $(0,2 \pi)$, extended $2 \pi$ periodically. From $y=0$ to $y=0+$ it has a jump $\frac{\pi}{2}$, so (2.7) has a jump $-\sin \alpha \cos \alpha$. Consequently by (2.5)

$$
\begin{aligned}
& G_{11}(0,0, \lambda)-\sin \alpha \cos \alpha=\frac{-1}{W(\lambda)} u_{1}(0, \lambda) v_{1}(0, \lambda) \\
& \quad=-\frac{\sin \alpha v_{1}(0, \lambda)}{\sin \alpha v_{2}(0, \lambda)+\cos \alpha v_{1}(0, \lambda)}=\frac{-\sin \alpha}{m(\lambda) \sin \alpha+\cos \alpha}
\end{aligned}
$$

as asserted.

\section{Lemma 2.2.}

$$
\begin{aligned}
G_{22}(0,0, \lambda) & =\cos ^{2} \alpha \cdot p \cdot v \cdot \int_{-\infty}^{\infty} \frac{d \rho(t)}{\lambda-t} \\
= & -\sin \alpha \cos \alpha+\frac{m(\lambda) \cos \alpha}{m(\lambda) \sin \alpha+\cos \alpha} .
\end{aligned}
$$

Proof. Repeating the above reduction process we get that $G_{22}$ has the same jump as the series

$$
\cos \alpha \cdot \text { p.v. } \sum_{n \neq 0} \frac{\cos (n y+a(y))}{-\pi n}=\cos \alpha \sin a(y) \frac{2}{\pi} \sum_{1}^{\infty} \frac{\sin n y}{n} .
$$

So this jump is $\sin \alpha \cos \alpha$ and then

$$
\begin{aligned}
G_{22}(0,0, \lambda)+\sin \alpha \cos \alpha & =\frac{\cos \alpha v_{2}(0, \lambda)}{\sin \alpha v_{2}(0, \lambda)+\cos \alpha v_{1}(0, \lambda)} \\
& =\frac{m(\lambda) \cos \alpha}{m(\lambda) \sin \alpha+\cos \alpha}
\end{aligned}
$$

and we are done.

Now the next statements follow immediately:

Corollary 2.3. a) If $\sin \alpha \neq 0$, then

$$
\text { p.v. } \int_{-\infty}^{\infty} \frac{d \rho(t)}{\lambda-t}=\cot \alpha-\frac{1}{\sin \alpha} \frac{1}{m(\lambda) \sin \alpha+\cos \alpha} .
$$

b) If $\cos \alpha \neq 0$, then

$$
p . v . \int_{-\infty}^{\infty} \frac{d \rho(t)}{\lambda-t}=-\tan \alpha+\frac{1}{\cos \alpha} \frac{m(\lambda)}{m(\lambda) \sin \alpha+\cos \alpha} .
$$

c)

$$
p . v . \int_{-\infty}^{\infty} \frac{d \rho(t)}{\lambda-t}=\frac{m(\lambda) \cos \alpha-\sin \alpha}{m(\lambda) \sin \alpha+\cos \alpha} .
$$

Corollary 2.4. The spectral function defines $\tan \alpha$ and the $m$-function, and conversely, the $m$-function and $\tan \alpha$ define the spectral function.

Proof. The spectral function determines the $\bmod \pi$ value of $\alpha$, i.e. $\tan \alpha$, hence by Corollary $2.3 \mathrm{c}$ ) it also defines $m(\lambda)$. Conversely, $m(\lambda)$ and $\tan \alpha$ define the transform p.v. $\int_{-\infty}^{\infty} \frac{d \rho(t)}{\lambda-t}$ and then they define $\rho(t)$ itself by the Stieltjes inversion formula, see [10]. 
Proof of Theorem 1.3. Define a boundary condition at $x=0$ with $\alpha=0$. Then by Corollary $2.3 \mathrm{~b}) m(\lambda)=\mathrm{p} . \mathrm{v} \cdot \int_{-\infty}^{\infty} \frac{d \rho(t)}{\lambda-t}$, and this defines $\rho$ by the inversion formula. From $\rho$ we determine $V$ and $\tan \beta$. We are done.

Next we analyze the growth properties of some infinite products in order to prove Theorems 1.8 and 1.9. Consider a sequence of arbitrary values

$$
\lambda_{n}=n+\mathbf{O}(1), \quad n \in \mathbb{Z}
$$

Let $S$ be a set of integers, almost symmetric with respect to the origin. This means that

$$
S \subset \mathbb{Z}, \quad n \in S \Rightarrow-n \in S
$$

with finitely many exceptions. Denote further

$$
n_{S}(t)=\left\{\begin{array}{ccc}
\sum_{0 \leqslant \lambda_{n} \leqslant t} 1 & \text { if } t>0 \\
-\sum_{t<\lambda_{n}<0} 1 & \text { if } t<0 .
\end{array}\right.
$$

Lemma 2.5. Suppose (2.8), (2.9) and that $\lambda_{n} \neq 0$ for $n \in S$. Then the product

$$
w_{S}(z)=p \cdot v \cdot \prod_{n \in S}\left(1-\frac{z}{\lambda_{n}}\right)
$$

converges locally uniformly and defines an entire function with zeros $\lambda_{n}$. Further we have

$$
\begin{aligned}
\ln \left|w_{S}(z)\right| & =p \cdot v \cdot \int_{-\infty}^{\infty} \frac{n_{S}(t)}{t} \frac{|z|^{2}-x t}{|z|^{2}-2 x t+t^{2}} \mathrm{~d} t \\
& =p \cdot v \cdot \int_{-\infty}^{\infty} \frac{n_{S}(t)}{t} \frac{y^{2}-x(t-x)}{y^{2}+(t-x)^{2}} \mathrm{~d} t
\end{aligned}
$$

where $z=x+i y$.

Proof. The locally uniform convergence of the product follows from the simple estimate

$$
\begin{aligned}
\left(1-\frac{z}{\lambda_{n}}\right)\left(1-\frac{z}{\lambda_{-n}}\right) & =1-z\left(\frac{1}{\lambda_{n}}+\frac{1}{\lambda_{-n}}\right)+\frac{z^{2}}{\lambda_{n} \lambda_{-n}} \\
& =1+\mathbf{O}\left(\frac{1}{n^{2}}\right) .
\end{aligned}
$$


To show (2.11) we integrate by parts

$$
\begin{aligned}
& \ln \left|w_{S}(z)\right|^{2}=\text { p.v. } \sum \ln \left|1-\frac{z}{\lambda_{n}}\right|^{2} \\
& =\text { p.v. } \sum \ln \left(\left(1-\frac{x}{\lambda_{n}}\right)^{2}+\frac{y^{2}}{\lambda_{n}^{2}}\right) \\
& =\text { p.v. } \sum \ln \left(1-\frac{2 x}{\lambda_{n}}+\frac{|z|^{2}}{\lambda_{n}^{2}}\right) \\
& =\operatorname{p.v} \cdot \int_{-\infty}^{\infty} \ln \left(1-\frac{2 x}{t}+\frac{|z|^{2}}{t^{2}}\right) \mathrm{d} n_{S}(t) \\
& =\text { p.v. }\left[\ln \left(1-\frac{2 x}{t}+\frac{|z|^{2}}{t^{2}}\right) n_{S}(t)\right]_{-\infty}^{\infty} \\
& \text { - p.v. } \int_{-\infty}^{\infty} n_{S}(t) \frac{\frac{2 x}{t^{2}}-\frac{2|z|^{2}}{t^{3}}}{1-\frac{2 x}{t}+\frac{|z|^{2}}{t^{2}}} \mathrm{~d} t .
\end{aligned}
$$

From

$$
\begin{aligned}
\ln \left(1-\frac{2 x}{t}+\frac{|z|^{2}}{t^{2}}\right) n_{S}(t) & =\left(-\frac{2 x}{t}+\frac{|z|^{2}}{t^{2}}\right) n_{S}(t)(1+\mathbf{o}(1)) \\
& =-2 x \frac{n_{S}(t)}{t}+\mathbf{o}(1) \quad(t \rightarrow \pm \infty)
\end{aligned}
$$

the almost symmetrical property of $S$ implies that

$$
\begin{aligned}
\ln \left|w_{S}(z)\right| & =\text { p.v. } \int_{-\infty}^{\infty} n_{S}(t) \frac{\frac{|z|^{2}}{t^{3}}-\frac{x}{t^{2}}}{1-\frac{2 x}{t}+\frac{|z|^{2}}{t^{2}}} \mathrm{~d} t \\
& =\text { p.v. } \int_{-\infty}^{\infty} \frac{n_{S}(t)}{t} \frac{|z|^{2}-x t}{t^{2}-2 x t+|z|^{2}} \mathrm{~d} t \\
& =\text { p.v. } \int_{-\infty}^{\infty} \frac{n_{S}(t)}{t} \frac{y^{2}-x(t-x)}{y^{2}+(t-x)^{2}} \mathrm{~d} t .
\end{aligned}
$$

Lemma 2.6. If we shift the zeros by $\mathbf{O}\left(\frac{1}{n}\right)$, the order of magnitude of $w_{S}$ remains the same, except near the zeros. In other words, if

$$
\lambda_{n}^{*}=\lambda_{n}+\mathbf{O}\left(\frac{1}{n}\right), \lambda_{n}^{*} \neq 0, w_{S}^{*}(z)=p \cdot v \cdot \prod_{n \in S}\left(1-\frac{z}{\lambda_{n}^{*}}\right),
$$

then for every $\delta>0$,

$$
\left|w_{S}^{*}(z)\right| \asymp\left|w_{S}(z)\right| \text { if }\left|z-\lambda_{n}\right|>\delta,\left|z-\lambda_{n}^{*}\right|>\delta \forall n .
$$

The notation $\asymp$ means that both $\left|\frac{w_{S}^{*}(z)}{w_{S}(z)}\right|$ and $\left|\frac{w_{S}(z)}{w_{S}^{*}(z)}\right|$ are bounded.

Proof. We have to show that

$$
\ln \left|w_{S}^{*}(z)\right|-\ln \left|w_{S}(z)\right|
$$

is bounded whenever $\left|z-\lambda_{n}\right|>\delta$ and $\left|z-\lambda_{n}^{*}\right|>\delta$. For bounded $z \ln \left|w_{S}^{*}(z)\right|$ and $\ln \left|w_{S}(z)\right|$ are both bounded, so we can suppose that $|z|$ is large, say, $|z| \geqslant K$. By 
the formula (2.11) we have

$$
\begin{gathered}
\ln \left|w_{S}^{*}(z)\right|-\ln \left|w_{S}(z)\right| \\
=\text { p.v. } \int_{-\infty}^{\infty} \frac{n_{S}^{*}(t)-n_{S}(t)}{t} \frac{y^{2}-x(t-x)}{y^{2}+(t-x)^{2}} \mathrm{~d} t .
\end{gathered}
$$

Here $\frac{n_{S}^{*}(t)-n_{S}(t)}{t}$ takes values of order $\mathbf{O}\left(\frac{1}{n}\right)$ in segments of length $\mathbf{O}\left(\frac{1}{n}\right)$, where $t=\mathbf{O}(n)$, otherwise it is zero. It implies first that the integral in (2.14) is convergent also in the ordinary sense. Secondly, $\frac{y^{2}}{y^{2}+(t-x)^{2}} \leqslant 1$ implies that

$$
\int_{-\infty}^{\infty} \frac{n_{S}^{*}(t)-n_{S}(t)}{t} \frac{y^{2}}{y^{2}+(t-x)^{2}} \mathrm{~d} t=\mathbf{O}\left(\sum \frac{1}{n^{2}}\right)=\mathbf{O}(1)
$$

uniformly in $z$ (we used the fact that $n_{S}(t)$ and $n_{S}^{*}(t)$ are zero near $t=0$ ). So it remains to prove that

$$
\begin{aligned}
& \int_{-\infty}^{\infty} \frac{n_{S}^{*}(t)-n_{S}(t)}{t} \frac{x(t-x)}{y^{2}+(t-x)^{2}} \mathrm{~d} t=\mathbf{O}(1) \\
& \quad\left(|z| \geqslant K,\left|z-\lambda_{n}\right|>\delta,\left|z-\lambda_{n}^{*}\right|>\delta\right)
\end{aligned}
$$

uniformly in $z$. If $x$ is bounded, then $y$ is large, so

$$
\frac{x(t-x)}{y^{2}+(t-x)^{2}}=\mathbf{O}\left(\frac{t-x}{1+(t-x)^{2}}\right)=\mathbf{O}(1)
$$

and then an estimate of the type (2.15) applies. Hence we can suppose that $x$ is large, e.g. $|x| \geqslant K / 2$. Consider only $x>K / 2$, since the case of negative $x$ is similar. Split the integral (2.16) into four parts as follows. If $t \geqslant \frac{3 x}{2}$, then $\frac{x(t-x)}{y^{2}+(t-x)^{2}} \asymp \frac{x t}{y^{2}+t^{2}}$, hence in (2.16)

$$
\left|\int_{\frac{3 x}{2}}^{\infty}\right| \leqslant c \sum_{k \geqslant \frac{3 x}{2}} \frac{1}{k^{2}} \frac{x k}{y^{2}+k^{2}} \leqslant c x \sum_{k \geqslant \frac{3 x}{2}} \frac{1}{k^{2}} \leqslant c
$$

uniformly in $|z|$. If $\frac{x}{2} \leqslant t \leqslant \frac{3 x}{2}$, then $y^{2}+(t-x)^{2}$ cannot be arbitrarily close to zero on the segments between $\lambda_{n}^{*}$ and $\lambda_{n}$, hence

$$
\frac{x(t-x)}{y^{2}+(t-x)^{2}}=\mathbf{O}\left(\frac{x|n-x|+x}{y^{2}+(n-x)^{2}+1}\right), \quad n=[t],
$$

and then in (2.16)

$$
\left|\int_{\frac{x}{2}}^{\frac{3 x}{2}}\right| \leqslant c \sum_{k=1}^{\left[\frac{x}{2}\right]} \frac{1}{x^{2}} \frac{k x}{y^{2}+k^{2}} \leqslant c \sum_{k=1}^{\left[\frac{x}{2}\right]} \frac{1}{y^{2}+k^{2}} \leqslant c .
$$

If $-\frac{x}{2} \leqslant t \leqslant \frac{x}{2}$, then $\frac{x(t-x)}{y^{2}+(t-x)^{2}} \asymp \frac{x^{2}}{y^{2}+x^{2}}=\mathbf{O}(1)$, hence $\left|\int_{-\frac{x}{2}}^{\frac{x}{2}}\right| \leqslant c$. Finally if $t \leqslant-\frac{x}{2}$, then $\frac{x(t-x)}{y^{2}+(t-x)^{2}} \asymp \frac{x t}{y^{2}+t^{2}}$, so in $(2.16)$

$$
\left|\int_{\infty}^{-\frac{x}{2}}\right| \leqslant c \sum_{k=\left[\frac{x}{2}\right]}^{\infty} \frac{1}{k^{2}} \frac{x k}{y^{2}+k^{2}} \leqslant c \sum \frac{1}{y^{2}+k^{2}} \leqslant c .
$$

This proves (2.16) and we are ready. 
Lemma 2.7. Under the conditions of Lemma 2.5 we have for fixed $x$

$$
\ln \left|w_{S}(z)\right|=\int_{-\infty}^{\infty} \frac{n_{S}(t)}{t} \frac{y^{2}}{y^{2}+t^{2}} \mathrm{~d} t+\mathbf{O}(1) \quad(|y| \rightarrow \infty) .
$$

The $\mathbf{O}$-term is locally uniform in $x$.

Proof. We have to transform (2.11) into (2.17). First,

$$
\begin{aligned}
& \left|\int_{-\infty}^{\infty} \frac{n_{S}(t)}{t}\left[\frac{y^{2}}{y^{2}+(t-x)^{2}}-\frac{y^{2}}{y^{2}+t^{2}}\right] \mathrm{d} t\right| \\
\leqslant c & \int_{-\infty}^{\infty} y^{2} \frac{x^{2}+2|t||x|}{\left(y^{2}+t^{2}\right)\left(y^{2}+(t-x)^{2}\right)} \mathrm{d} t
\end{aligned}
$$

and here

$$
\begin{gathered}
\int_{-\infty}^{\infty} \frac{y^{2} x^{2}}{\left(y^{2}+t^{2}\right)\left(y^{2}+(t-x)^{2}\right)} \mathrm{d} t \leqslant x^{2} \int_{-\infty}^{\infty} \frac{\mathrm{d} t}{y^{2}+t^{2}}=\mathbf{O}(1), \\
\int_{|t| \leqslant|y|} \frac{y^{2}|t||x|}{\left(y^{2}+t^{2}\right)\left(y^{2}+(t-x)^{2}\right)} \mathrm{d} t \leqslant|x| \int_{|t| \leqslant|y|} \frac{|t|}{y^{2}+t^{2}} \mathrm{~d} t=\mathbf{O}(1), \\
\int_{|t| \geqslant|y|} \frac{y^{2}|t||x|}{\left(y^{2}+t^{2}\right)\left(y^{2}+(t-x)^{2}\right)} \mathrm{d} t \leqslant c y^{2}|x| \int_{|t| \geqslant|y|} \frac{|t|}{t^{4}} \mathrm{~d} t=\mathbf{O}(1) .
\end{gathered}
$$

So it remains to show that

$$
\text { p.v. } \int_{-\infty}^{\infty} \frac{n_{S}(t)}{t} \frac{t-x}{y^{2}+(t-x)^{2}} \mathrm{~d} t=\mathbf{O}(1) \quad(|y| \rightarrow \infty)
$$

locally uniformly in $x$. From (2.8) and (2.9) we see that

$$
n_{S}(-t)=-n_{S}(t)+\mathbf{O}(1) \text {. }
$$

Taking into account that $\lambda_{n} \neq 0$, we get

$$
\begin{aligned}
& \text { p.v. } \int_{-\infty}^{\infty} \frac{n_{S}(t)}{t} \frac{t-x}{y^{2}+(t-x)^{2}} \mathrm{~d} t \\
= & \int_{\delta}^{\infty} \frac{n_{S}(t)}{t}\left[\frac{t-x}{y^{2}+(t-x)^{2}}-\frac{t+x}{y^{2}+(t+x)^{2}}\right] \mathrm{d} t \\
& +\mathbf{O}\left(\int_{\delta}^{\infty} \frac{1}{t} \frac{|t+x|}{y^{2}+(t+x)^{2}} \mathrm{~d} t\right) .
\end{aligned}
$$

Now we have

$$
\int_{\delta}^{\infty} \frac{1}{t} \frac{|t+x|}{y^{2}+(t+x)^{2}} \mathrm{~d} t \leqslant c \int_{\delta}^{\infty} \frac{\mathrm{d} t}{y^{2}+(t+x)^{2}}=\mathbf{O}(1) .
$$

Furthermore

$$
\begin{aligned}
& \frac{t-x}{y^{2}+(t-x)^{2}}-\frac{t+x}{y^{2}+(t+x)^{2}} \\
= & \frac{-2 x y^{2}+(t-x)(t+x) 2 x}{\left[y^{2}+(t-x)^{2}\right]\left[y^{2}+(t+x)^{2}\right]} \\
= & \mathbf{O}\left(\frac{1}{y^{2}+(t+x)^{2}}\right)
\end{aligned}
$$


and then

$$
\begin{gathered}
\int_{\delta}^{\infty} \frac{n_{S}(t)}{t}\left[\frac{t-x}{y^{2}+(t-x)^{2}}-\frac{t+x}{y^{2}+(t+x)^{2}}\right] \mathrm{d} t \\
=\mathbf{O}\left(\int_{\delta}^{\infty} \frac{\mathrm{d} t}{y^{2}+(t+x)^{2}}\right)=\mathbf{O}(1) .
\end{gathered}
$$

Finally we need a classical estimate of Levinson and a Phragmén-Lindelöf-type result:

Lemma 2.8 (Levinson [8]). Let $z_{n}, n \geqslant 1$, be complex numbers with

$$
\lim _{n \rightarrow \infty} \frac{n}{z_{n}}=D \in \mathbb{R}
$$

Suppose further that for some $c>0$

$$
\left|z_{n}-z_{m}\right| \geqslant c|n-m|
$$

Let

$$
F(z)=\prod_{1}^{\infty}\left(1-\frac{z^{2}}{z_{n}^{2}}\right)
$$

then for any $\varepsilon>0$, as $r=|z| \rightarrow \infty$

$$
F\left(r e^{i \varphi}\right)=\mathbf{O}\left(e^{\pi D r|\sin \varphi|+\varepsilon r}\right)
$$

and

$$
\frac{1}{F\left(r e^{i \varphi}\right)}=\mathbf{O}\left(e^{-\pi D r|\sin \varphi|+\varepsilon r}\right) \quad \text { if }\left|r e^{i \varphi}-z_{n}\right| \geqslant \frac{1}{8} c .
$$

Lemma 2.9 (Levin [11]). Let $F(z)$ be an entire function of zero exponential type i.e.

$$
\limsup _{r \rightarrow \infty} \frac{\ln M(r)}{r} \leqslant 0, \quad M(r)=\max _{\varphi}\left|F\left(r e^{i \varphi}\right)\right| .
$$

If $F(z)$ is bounded along a line, then $F(z)$ is constant. In particular, if $F(z) \rightarrow 0$ when $|z| \rightarrow \infty$ along a line then $F(z) \equiv 0$.

Proof of Theorem 1.9. Step 1. Introduce the function

$$
\begin{gathered}
F(z)=\frac{v_{1}(a, z) v_{2}^{*}(a, z)-v_{1}^{*}(a, z) v_{2}(a, z)}{\prod_{j=1}^{N} p_{j}(z)} \\
\text { where } p_{j}(z)=\text { p.v. } \prod_{n \in S_{j}}\left(1-\frac{z}{\lambda_{n}^{(j)}}\right) .
\end{gathered}
$$

If $\lambda_{n}^{(j)}=0$ we write $z$ instead of $1-\frac{z}{\lambda_{n}^{(j)}}$. The denominator of $F(z)$ has only simple zeros since the eigenvalues $\lambda_{n}^{(j)}$ are generated with a fixed right boundary condition. The values $\lambda_{n}^{(j)}, n \in S_{j}$, are zeros of the numerator, too, so $F(z)$ is an entire function. Indeed, denote by $\left(u_{1, j}(x, \lambda), u_{2, j}(x, \lambda)\right)$ the solution of $(2.1)$ with

$$
u_{1, j}(0, \lambda)=\sin \alpha_{j}, \quad u_{2, j}(0, \lambda)=-\cos \alpha_{j} .
$$


Since $V^{*}=V$ on $[0, a]$,

$$
u_{1, j}^{*}(a, \lambda)=u_{1, j}(a, \lambda), \quad u_{2, j}^{*}(a, \lambda)=u_{2, j}(a, \lambda) .
$$

Since $\lambda_{n}^{(j)}=\lambda_{n}^{(j)^{*}}$ is an eigenvalue, the vectors

$$
\left(u_{1, j}\left(x, \lambda_{n}^{(j)}\right), u_{2, j}\left(x, \lambda_{n}^{(j)}\right)\right) \quad \text { and } \quad\left(v_{1}\left(x, \lambda_{n}^{(j)}\right), v_{2}\left(x, \lambda_{n}^{(j)}\right)\right),
$$

on the one hand, and

$$
\left(u_{1, j}^{*}\left(x, \lambda_{n}^{(j)}\right), u_{2, j}^{*}\left(x, \lambda_{n}^{(j)}\right)\right) \quad \text { and } \quad\left(v_{1}^{*}\left(x, \lambda_{n}^{(j)}\right), v_{2}^{*}\left(x, \lambda_{n}^{(j)}\right)\right),
$$

on the other hand, are parallel. Hence

$$
\frac{v_{2}^{*}\left(a, \lambda_{n}^{(j)}\right)}{v_{1}^{*}\left(a, \lambda_{n}^{(j)}\right)}=\frac{u_{2, j}^{*}\left(a, \lambda_{n}^{(j)}\right)}{u_{1, j}^{*}\left(a, \lambda_{n}^{(j)}\right)}=\frac{u_{2, j}\left(a, \lambda_{n}^{(j)}\right)}{u_{1, j}\left(a, \lambda_{n}^{(j)}\right)}=\frac{v_{2}\left(a, \lambda_{n}^{(j)}\right)}{v_{1}\left(a, \lambda_{n}^{(j)}\right)} .
$$

So $F(z)$ is an entire function indeed.

Step 2. We estimate the numerator of $F(z)$ using (2.3), (2.4):

$$
\begin{aligned}
& v_{1}(a, z) v_{2}^{*}(a, z)-v_{1}^{*}(a, z) v_{2}(a, z) \\
= & \sin \left(z(\pi-a)-\int_{a}^{\pi} V-\beta\right) \cos \left(z(\pi-a)-\int_{a}^{\pi} V^{*}-\beta\right) \\
& -\sin \left(z(\pi-a)-\int_{a}^{\pi} V^{*}-\beta\right) \cos \left(z(\pi-a)-\int_{a}^{\pi} V-\beta\right) \\
& +\mathbf{O}\left(\frac{e^{2|\Im z|(\pi-a)}}{|z|}\right) \\
= & \sin \int_{a}^{\pi}\left(V^{*}-V\right)+\mathbf{O}\left(\frac{e^{2|\Im z|(\pi-a)}}{|z|}\right) .
\end{aligned}
$$

From $a<\pi$ we know that this function has infinitely many real zeros and the zeros are not bounded. This is compatible with the above estimate only when

$$
\sin \int_{a}^{\pi}\left(V^{*}-V\right)=0
$$

and then

$$
v_{1}(a, z) v_{2}^{*}(a, z)-v_{1}^{*}(a, z) v_{2}(a, z)=\mathbf{O}\left(\frac{e^{2|\Im z|(\pi-a)}}{|z|}\right) .
$$

Step 3. We estimate the denominator of $F(z)$. By Lemma 2.6

$$
\left|p_{j}(z)\right| \asymp\left|\widehat{p}_{j}(z)\right| \text { if }\left|z_{n}^{(j)}\right| \geqslant \delta,\left|z-n-\frac{\vartheta_{j}}{\pi}\right| \geqslant \delta \text { for } n \in S_{j},
$$

where

$$
\widehat{p}_{j}(z)=\text { p.v. } \prod_{n \in S_{j}}\left(1-\frac{z}{n+\frac{\vartheta_{j}}{\pi}}\right), \quad \vartheta_{j}=\beta-\alpha_{j}+\int_{0}^{\pi} V .
$$



Again, if $n+\frac{\vartheta_{j}}{\pi}=0$, we substitute $1-\frac{z}{n+\frac{\vartheta_{j}}{\pi}}$ by $z$. In calculating $\widehat{p}_{j}\left(z+\frac{\vartheta_{j}}{\pi}\right)$ we
use

$$
1-\frac{z+\frac{\vartheta_{j}}{\pi}}{n+\frac{\vartheta_{j}}{\pi}}=\frac{n-z}{n+\frac{\vartheta_{j}}{\pi}}=\left(1-\frac{z}{n}\right) \frac{n}{n+\frac{\vartheta_{j}}{\pi}}=\left(1-\frac{z}{n}\right)\left(1-\frac{\frac{\vartheta_{j}}{\pi}}{n+\frac{\vartheta_{j}}{\pi}}\right) .
$$

to obtain

$$
\widehat{p}_{j}\left(z+\frac{\vartheta_{j}}{\pi}\right)=\widetilde{p}_{j}(z) \cdot c_{j}, \quad \widetilde{p}_{j}(z)=\text { p.v. } \prod_{n \in S_{j}}\left(1-\frac{z}{n}\right) .
$$

We handle the case $n=0 \in S_{j}$ as above. Arrange the values $n \in S_{j}$ in an increasing sequence $z_{k}$. Since $n_{j}\left(z_{k}\right)=k+\mathbf{O}(1)$, we have

$$
\frac{k}{z_{k}}=\frac{n_{j}\left(z_{k}\right)}{z_{k}}+\mathbf{o}(1) \longrightarrow \gamma_{j} \quad(k \rightarrow \infty)
$$

Now the almost symmetric property of $S_{j}$ implies a lower estimate by Lemma 2.8 : for every $\varepsilon>0$ there exists a $c=c(\varepsilon, \delta)>0$ such that

$$
\left|\widetilde{p}_{j}(z)\right| \geqslant c e^{\pi \gamma_{j}|\Im z|-\varepsilon|z|} \quad \text { if } \quad|z-n| \geqslant \delta \quad \text { for } n \in S_{j} .
$$

(If the product $\widetilde{p}_{j}$ is finite, this estimate follows immediately.) By the above considerations

$$
\left|p_{j}(z)\right| \asymp\left|\widehat{p}_{j}(z)\right| \asymp\left|\widetilde{p}_{j}\left(z-\frac{\vartheta_{j}}{\pi}\right)\right| \geqslant c e^{\pi \gamma_{j}|\Im z|-2 \varepsilon|z|}
$$

for $|z|$ large enough, if $\left|z-n-\frac{\vartheta_{j}}{\pi}\right| \geqslant \delta,\left|z-\lambda_{n}^{(j)}\right| \geqslant \delta$ hold for $n \in S_{j}$. Hence the whole denominator of $F$ has a lower estimate

$$
\prod_{j=1}^{N}\left|p_{j}(z)\right| \geqslant c e^{\pi \sum_{j} \gamma_{j}|\Im z|-2 \varepsilon N|z|} \geqslant c e^{2(\pi-a)|\Im z|-2 \varepsilon N|z|}
$$

By Step 2 this means that

$$
\begin{aligned}
& |F(z)| \leqslant c e^{(2 N+1) \varepsilon|z|} \text { for }|z| \text { large enough, if }\left|z-n-\frac{\vartheta_{j}}{\pi}\right| \geqslant \delta, \\
& \qquad\left|z-\lambda_{n}^{(j)}\right| \geqslant \delta \text { hold for } n \in S_{j} .
\end{aligned}
$$

Here $\varepsilon>0$ is arbitrary and $\delta>0$ can be chosen so small that the excluded circles of radius $\delta$ are disjoint for large $|n|$. Consequently the maximum modulus principle shows that

$$
|F(z)| \leqslant c e^{(2 N+1) \varepsilon|z|}, \quad z \in \mathbb{C} .
$$

This means that $F(z)$ is of zero exponential type.

Step 4. We show that

$$
F(i y) \rightarrow 0 \quad \text { for } \quad|y| \rightarrow \infty .
$$

In Step 3 we proved that

$$
\left|p_{j}(i y)\right| \asymp\left|\widehat{p}_{j}(i y)\right| \asymp\left|\widetilde{p}_{j}\left(i y-\frac{\vartheta_{j}}{\pi}\right)\right|, \quad|y| \rightarrow \infty .
$$

Furthermore, Lemma 2.7 states

$$
\left|\widetilde{p}_{j}\left(i y-\frac{\vartheta_{j}}{\pi}\right)\right| \asymp\left|\widetilde{p}_{j}(i y)\right|, \quad|y| \rightarrow \infty .
$$


Consequently the denominator of $F$ can be estimated as follows

$$
\prod_{j=1}^{N}\left|p_{j}(i y)\right| \asymp|y|^{d} \exp \left(\int_{-\infty}^{\infty} \frac{\sum \widetilde{n}_{j}(t)}{t} \frac{y^{2}}{y^{2}+t^{2}} \mathrm{~d} t\right)
$$

if $0 \in S_{j}$ is fulfilled $d$ times. Now we have

$$
\begin{array}{r}
\int_{1}^{\infty} \frac{[t]}{t} \frac{y^{2}}{y^{2}+t^{2}} \mathrm{~d} t+\int_{-\infty}^{-1} \frac{-[-t]}{t} \frac{y^{2}}{y^{2}+t^{2}} \mathrm{~d} t \\
=\ln \left|\frac{\sin (\pi i y)}{\pi i y}\right|=\pi|y|-\ln |y|+\mathbf{O}(1) ;
\end{array}
$$

because of the known formula

$$
\frac{\sin \pi z}{\pi z}=\prod_{n=1}^{\infty}\left(1-\frac{z^{2}}{n^{2}}\right)
$$

and (2.11). On the other hand

$$
\begin{gathered}
\int_{1}^{\infty} \frac{1}{t} \frac{y^{2}}{y^{2}+t^{2}} \mathrm{~d} t=\int_{1}^{\infty}\left(\frac{1}{t}-\frac{t}{y^{2}+t^{2}}\right) \mathrm{d} t \\
\quad=\frac{1}{2} \ln \left(y^{2}+1\right)=\ln |y|+\mathbf{O}(1),
\end{gathered}
$$

and analogously

$$
\int_{-\infty}^{-1} \frac{1}{t} \frac{y^{2}}{y^{2}+t^{2}} \mathrm{~d} t=-\ln |y|+\mathbf{O}(1) .
$$

From inequality (1.19) we infer

$$
\begin{aligned}
& \int_{-\infty}^{\infty} \frac{\sum \widetilde{n}_{j}(t)}{t} \frac{y^{2}}{y^{1}+t^{2}} \mathrm{~d} t \geqslant 2(\pi-a)|y| \\
& +\left(-2\left(1-\frac{a}{\pi}\right)+\left(2\left(1-\frac{a}{\pi}\right)-d+\mu-1+\varepsilon\right)-\mu\right) \ln |y|+\mathbf{O}(1) \\
= & 2(\pi-a)|y|+(-1+\varepsilon-d) \ln |y|+\mathbf{O}(1)
\end{aligned}
$$

and then

$$
\prod_{j=1}^{N}\left|p_{j}(i y)\right| \geqslant c|y|^{-1+\varepsilon} e^{2(\pi-a)|y|} \quad(|y| \rightarrow \infty) .
$$

By Step 2

$$
\left|v_{1}(a, i y) v_{2}^{*}(a, i y)-v_{1}^{*}(a, i y) v_{2}(a, i y)\right| \leqslant c \frac{e^{2(\pi-a)|y|}}{|y|}
$$

and then

$$
F(i y)=\mathbf{O}\left(|y|^{-\epsilon}\right) \rightarrow 0, \quad|y| \rightarrow \infty .
$$

Now Lemma 2.9 implies

$$
F(z) \equiv 0
$$

or

$$
\frac{v_{2}(a, z)}{v_{1}(a, z)} \equiv \frac{v_{2}^{*}(a, z)}{v_{1}^{*}(a, z)}
$$


From $V^{*}=V$ on $[0, a]$ it follows that

$$
m(z)=\frac{v_{2}(0, z)}{v_{1}(0, z)}=\frac{v_{2}^{*}(0, z)}{v_{1}^{*}(0, z)}=m^{*}(z) .
$$

By Theorem 1.3 this implies $V^{*}=V$ on $[0, \pi]$.

Proof of Theorem 1.8. It is analogous to the above proof, so we only mention the main points. Since infinitely many eigenvalues of $q$ and of $q^{*}$ are the same, from a well-known eigenfunction asymptotics (see e.g. [10], Ch. I) we get that

$$
\int_{0}^{\pi}\left(q^{*}-q\right)=0
$$

Again by the standard eigenfunction asymptotics method of [10] we get for $\sin \beta \neq 0$

$$
\begin{aligned}
v(x, \lambda)=\sin \beta \cos s(\pi-x) & +\left(\cos \beta+\sin \beta \cdot \int_{x}^{\pi} \frac{q}{2}\right) \frac{\sin s(\pi-x)}{s} \\
& +\mathbf{o}\left(\frac{e^{|\Im s|(\pi-x)}}{|s|}\right), \\
v^{\prime}(x, \lambda)=\sin \beta \cdot s \cdot \sin s(\pi-x) & -\left(\cos \beta+\sin \beta \cdot \int_{x}^{\pi} \frac{q}{2}\right) \cos s(\pi-x) \\
& +\mathbf{o}\left(e^{|\Im s|(\pi-x)}\right),
\end{aligned}
$$

where $\lambda=s^{2}$ and $s \rightarrow \infty$ along any ray $\arg s=$ const. Analogously, for $\sin \beta=0$

$$
\begin{aligned}
v(x, \lambda) & =\cos \beta\left(\frac{\sin s(\pi-x)}{s}-\frac{\cos s(\pi-x)}{s^{2}} \int_{x}^{\pi} \frac{q}{2}\right) \\
& +\mathbf{o}\left(\frac{e^{|\Im s|(\pi-x)}}{|s|^{2}}\right), \\
v^{\prime}(x, \lambda) & =-\cos \beta\left(\cos s(\pi-x)+\frac{\sin s(\pi-x)}{s} \int_{x}^{\pi} \frac{q}{2}\right) \\
& +\mathbf{o}\left(\frac{e^{|\Im s|(\pi-x)}}{|s|}\right) .
\end{aligned}
$$

Combining this with (2.24), we finally obtain

$$
\begin{gathered}
v(a, z) v^{*^{\prime}}(a, z)-v^{*}(a, z) v^{\prime}(a, z) \\
=\left\{\begin{array}{lll}
\mathbf{o}\left(e^{2|\Im \sqrt{z}|(\pi-a)}\right) & \text { if } & \sin \beta \neq 0, \\
\mathbf{o}\left(\frac{e^{2|\Im \sqrt{z}|(\pi-a)}}{|z|}\right) & \text { if } & \sin \beta=0 .
\end{array}\right.
\end{gathered}
$$

The denominator of $F(z)$ can be estimated by

$$
\ln \left|\prod_{j=1}^{N} p_{j}(i y)\right|=\int_{1}^{\infty} \frac{\sum_{j=1}^{N} n_{j}(\sqrt{t})}{t} \frac{y^{2}}{y^{2}+t^{2}} \mathrm{~d} t+\mathbf{O}(1) .
$$


Now

$$
\begin{aligned}
& \int_{1}^{\infty} \frac{[\sqrt{t}]}{t} \frac{y^{2}}{y^{2}+t^{2}} \mathrm{~d} t=\ln \left|\prod_{n=1}^{\infty}\left(1-\frac{i y}{n^{2}}\right)\right|+\mathbf{O}(1) \\
= & \ln \left|\frac{\sin \pi \sqrt{i y}}{\pi \sqrt{i y}}\right|+\mathbf{O}(1)=\frac{\pi}{\sqrt{2}} \sqrt{|y|}-\frac{1}{2} \ln |y|+\mathbf{O}(1), \\
& \int_{1}^{\infty} \frac{\left[\sqrt{t}+\frac{1}{2}\right]}{t} \frac{y^{2}}{y^{2}+t^{2}} \mathrm{~d} t=\ln \left|\prod_{n=0}^{\infty}\left(1-\frac{i y}{\left(n+\frac{1}{2}\right)^{2}}\right)\right|+\mathbf{O}(1) \\
= & \ln |\cos \pi \sqrt{i y}|+\mathbf{O}(1)=\frac{\pi}{\sqrt{2}} \sqrt{|y|}+\mathbf{O}(1) .
\end{aligned}
$$

So by (1.17) in the case $\sin \beta \neq 0$ we obtain that

$$
\ln \left|\prod_{j=1}^{N} p_{j}(i y)\right| \geqslant 2(\pi-a) \sqrt{\frac{|y|}{2}}+\mathbf{O}(1)
$$

which means by $(2.25)$ that

$$
F(i y) \rightarrow 0 \quad(y \rightarrow \infty)
$$

If $\sin \beta=0$, then similarly

$$
\ln \left|\prod_{j-1}^{N} p_{j}(i y)\right| \geqslant 2(\pi-a) \sqrt{\frac{|y|}{2}}-\ln |y|+\mathbf{O}(1)
$$

and from $(2.25)$ we get $(2.26)$. Since $(2.26)$ implies $F(z) \equiv 0$, we are ready.

\section{REFERENCES}

1. G. Borg, Eine Umkehrung der Sturm-Liouvilleschen Eigenwertaufgabe, Acta Math. 78 (1946), 1-96. MR 7:382d

2. G. Borg, Uniqueness theorems in the spectral theory of $y^{\prime \prime}+(\lambda-q(x)) y=0$, Proc. 11th Scandinavian Congress of Mathematicians, Johan Grundt Tanums Forlag, Oslo, 1952, pp. 276-287. MR 15:315a

3. F. Gesztesy, R. del Rio and B. Simon, Inverse spectral analysis with partial information on the potential, III. Updating boundary conditions, Intl. Math. Research Notices 15 (1997), 751-758. MR 99a:34032

4. F. Gesztesy, R. del Rio and B. Simon, Corrections and Addendum to "Inverse spectral analysis with partial information on the potential, III. Updating boundary conditions", Intl. Math. Research Notices 11 (1999), 623-625. MR 2000d:34025

5. F. Gesztesy and B. Simon, Inverse spectral analysis with partial information on the potential, II. The case of discrete spectrum, Trans. Amer. Math. Soc. 352 (2000), 2765-2787. MR 2000j:34019

6. F. Gesztesy and B. Simon, On the determination of the potential from three spectra, Trans. Amer. Math. Soc. 189(2) (1999), 85-92. MR 2000i:34026

7. H. Hochstadt and B. Lieberman, An inverse Sturm-Liouville problem with mixed given data, SIAM J. Appl. Math. 34 (1978), 676-680. MR 57:10077

8. N. Levinson, Gap and density theorems, AMS Coll. Publ., 1940, New York. MR 2:180d

9. B. M. Levitan and I. S. Sargsjan, Sturm-Liouville and Dirac operators (in Russian), Nauka, Moscow 1988; English transl. MR 92i:34119

10. B. M. Levitan and I. S. Sargsjan, Introduction to spectral theory (in Russian), Nauka, Moscow 1970. MR 33:4362 
11. B. Ja. Levin, Distribution of zeros of entire functions (in Russian), GITTL, Moscow 1956. MR 19:402c

12. V.A. Marchenko, Certain problems in the theory of second order differential operators (in Russian), Dokl. Akad. Nauk. SSSR, 72 (1950), 457-460.

13. B.A. Watson, Inverse spectral problems for weighted Dirac systems, Inverse Problems, 15(3) (1999), 793-805. MR 2000d:34184

Budapest University of Technology and Economics, Institute of Mathematics, H 1111 Budapest, MÜegyetem RKP. 3-9, Hungary

E-mail address: horvath@math.bme.hu 